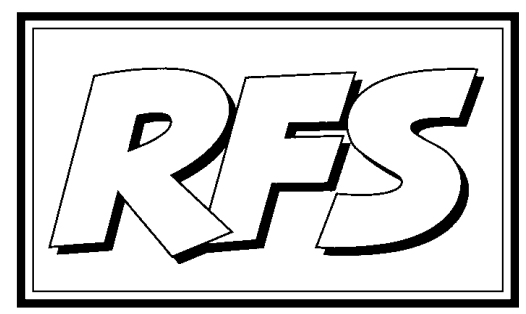

Revista de Fomento Social, 56 (2001), 485-497

\title{
Iniciativa por el desarrollo rural de Nicaragua*
}

\author{
“BASES PARA UN PLAN DE DESARROLLO RURAL DE NICARAGUA. Una \\ Propuesta para la Discusión y la Acción"
}

\section{¿Qué es la iniciativa?}

La Iniciativa por el Desarrollo Rural de Nicaragua es un proceso participativo de reflexión y debate con vocación propositiva y de incidencia, centrado en la problemática del medio rural del país y en la elaboración de marcos, estrategias y propuestas adecuadas para su desarrollo humano y sostenible.

Inicialmente articulada a través de un grupo de reflexión que se denominó Grupo de Tepeyac, actualmente la Iniciativa es una institución no formal más amplia que trasciende al mencionado Grupo y que configura un movimiento en el que, desde la búsqueda del interés común y la renuncia a intereses

* El presente texto resume el proceso de elaboración de una política de desarrollo rural para Nicaragua en el que ha participado, entre otros, un equipo de ETEA (como se explica en el propio documento). Este documento, resumen de otro más amplio, fue presentado en Managua el pasado mes de septiembre. 
partidistas, personales o estrictamente institucionales, han participado hasta la fecha más de 100 personas vinculadas al desarrollo rural a través de su participación en diferentes organismos públicos, privados, universidades y agencias de cooperación internacional.

A pesar de las dificultades de diversa índole encontradas a lo largo de su recorrido, el movimiento de la Iniciativa constituye un ejercicio de auténtica cooperación o trabajo conjunto en sus diversas dimensiones: cooperación interinstitucional, cooperación interuniversitaria y cooperación internacional.

El documento "Bases para un Plan de Desarrollo Rural de Nicaragua. Una propuesta para la discusión y la acción" es el resultado de este esfuerzo abierto y participativo de reflexión sobre los problemas del medio rural. Este trabajo propone un marco conceptual y una visión de desarrollo rural de Nicaragua, analiza las causas de los problemas y propone estrategias consensuadas de actuación.

\section{La historia. ¿Quiénes han participado en el proceso?}

El Grupo de Tepeyac (GT) seconstituyó en junio de 1999 en el contexto del proyecto Iniciativa por el Desarrollo Rural de Nicaragua (financiado por la Agencia Española de Cooperación Internacional (AECI), con el impulso del Ministerio Agropecuario y Forestal de Nicaragua (MAGFOR), el Programa de Naciones Unidas para el Desarrollo (PNUD), la propia Agencia Española de Cooperación Internacional (AECI), la Universidad Centroamericana (UCA), ETEA (Institución Universitaria de la Compañía de Jesús, adscrita a la Universidad de Córdoba) y el Equipo de Desarrollo Rural de la Universidad de Córdoba. Posteriormente se amplió la participación de universidades españolas con la incorporación del Grupo de Estudios y Promoción del Desarrollo del Departamento de Proyectos de Ingeniería de la Universidad Politécnica de Valencia, conformando el actual Equipo interuniversitario de Córdoba (España).

El Grupo de Tepeyac nació como foro permanente de discusión y reflexión sobre desarrollo rural, con vocación interdisciplinar e interinstitucional, formado por técnicos implicados en el tema. La capacidad y potencialidad de este foro de debate queda de manifiesto por la diversidad de sus componentes y por el amplio abanico de campos en los que aplican su experiencia. 
En el acto de constitución le fue encargado al GT, como primer cometido, la elaboración de unas Bases para el Desarrollo Rural de Nicaragua que facilitase los procesos de planificación y elaboración de políticas con el máximo consenso y participación. Para responder a este mandato la composición inicial del Grupo fue ampliada incorporándose la participación de otras instituciones, entre las que destacan el Instituto Nicaragüense de Fomento Municipal (INIFOM), el Instituto de Desarrollo Rural (IDR), la Unión Nacional de Agricultores y Ganaderos (UNAG), la Unión de Productores Agropecuarios de Nicaragua (UPANIC), la Unidad de Estrategias para la Seguridad Alimentaria (UESA, UE), la Organización de las Naciones Unidas para la Agricultura y la Alimentación (FAO) y el Instituto Interamericano de Cooperación para la Agricultura (IICA),

Los trabajos para la elaboración de las Bases han contado con sesiones plenarias de reflexión y debate colectivo y con comisiones temáticas de trabajo constituidas ad hoc entre las instituciones participantes. Los trabajos de dichas comisiones concluyen con la celebración, en diciembre de 2000, de talleres temáticos abiertos a la participación tanto del resto deintegrantes de las comisiones como a otras personas interesadas.

Tras casi dos años de trabajos sobre el tema se celebró en mayo de 2001, en Selva Negra (Matagalpa), un taller en el que participaron cerca de 50 personas representantes de todas las instituciones significativas que habían intervenido de una u otra forma en los trabajos del Grupo de Tepeyac a lo largo de su historia, así como algunos expertos invitados a título personal. Este evento fue completado con un segundo taller que tuvo lugar en el Instituto Nicaragüense de Cultura Hispánica el 23 de julio pasado. Ambos talleres se programaron como el último paso de reflexión, debate y consenso de los colectivos, instituciones y expertos del Grupo, previo a someter a la opinión pública su resultado y propuestas.

El Taller de Selva Negra concluyó con la constatación de que la Iniciativa es en la actualidad un movimiento más amplio que trasciendeal GT . Concluyó asimismo con el acuerdo de terminar el Documento de Bases que ahora se presenta, a partir de los insumos existentes y de la sistematización de los aportes realizados en el propio taller, y de avanzar en el proceso de concertación, tanto para lograr el mayor número posible de adhesiones institucionales y personales al proceso, como para continuar en el itinerario político adecuado que conduzca en un futuro próximo a la deseable elaboración de una Ley Nacional de Desarrollo Rural. 


\section{¿Qué se pretende con la iniciativa? ¿Qué se propone en esencia?}

- Crear las condiciones necesarias para facilitar el diseño y aplicación de una política integral de desarrollo rural elaborada y ejecutada con la participación del mayor número de agentes implicados en el medio rural de Nicaragua.

- Consensuar el concepto, los objetivos y el modo de poner en marcha una estrategia nacional de desarrollo rural.

- Contribuir a la elaboración participativa de una metodología nicaragüense de análisis, diagnóstico y diseño de estrategias de desarrollo rural.

- Contribuir a la elaboración de un Plan Nacional de Desarrollo Rural que ofrezca a la sociedad nicaragüense e internacional un esquema de objetivos e instrumentos coherente y adaptado a las necesidades de Nicaragua.

- Avanzar en el diseño de una metodología de acción territorial acorde con la filosofía inspiradora de las Bases.

\section{¿A qué meta aspira?}

La meta del proceso es la siguiente:

Existe para finales de 2002 una Política Nacional de Desarrollo Rural consensuada:

$\checkmark$ una política de Estado que incorpore un Plan Nacional de Desarrollo Rural a largo plazo;

$\checkmark$ nacida de la concertación interinstitucional pública y privada;

$\checkmark$ que incluya en su formulación la territorialización de las discusiones y aportaciones, así como la participación de los habitantes rurales en el proceso.

Una política que sea refrendada en la Asamblea Nacional mediante la promulgación de una Ley de Desarrollo Rural:

$\checkmark$ que articule la institucionalidad necesaria para implementar el modelo de desarrollo rural consensuado; 
que contenga la definición de los instrumentos necesarios para su efectiva aplicación.

Todo ello, sin perder de vista la urgencia de pensar en el largo plazo y siendo conscientes de que, en coherencia con lo manifestado en este documento, el propio proceso de generación de la Política, Plan y Ley es tanto o más importante que los mismos.

\section{¿Cuál es el contenido de las bases?}

Las Bases suponen un esfuerzo de debate, desde el respeto a la pluralidad, que permita, con el necesario rigor metodológico, definir y proponer acciones de desarrollo rural.

Aparte de una Introducción (Capítulo Primero) queexplica los antecedentes y objetivos, la génesis y el proceso de su elaboración, las Bases comprenden los siguientes capítulos:

El Capítulo Segundo (Principios Generales) tiene por objetivo establecer los principios básicos (tanto de índole general como los más específicos del desarrollo rural) que deben inspirar cualquier Plan de Desarrollo Rural, ya sea en el ámbito nacional o local. En torno a estos principios se ha llegado a un elevado grado de acuerdo y, a pesar de su aparente carácter teórico, su aceptación generalizada constituye un avance considerable de cara a la consecución logros concretos en la planificación del desarrollo rural de Nicaragua.

En el Capítulo Tercero se aborda el Diagnóstico General de la problemática rural nicaragüense. Es un capítulo de síntesis, resultado del resumen de una serie de insumos previos más extensos, procedentes a su vez en su mayor parte del trabajo de las Comisiones Temáticas del Grupo de Tepeyac y de algunas otras aportaciones individuales. Más que en sus detalles, existe amplio consenso en la estructura de análisis seguido que ha subdividido la materia en tres grandes bloques o temas:

\section{Temas "transversales"}

I.1 En busca del "buen gobierno": institucionalidad nacional y local del desarrollo rural

I.2 La dialéctica "macro"-“micro". Interdependencia de las políticas 
macrofinancieras y las sectoriales

I.3 El manejo del patrimonio natural. Gestión de los ecosistemas

I.4 Problemática de género y potencialidad de la mujer rural

\section{Temas "sectoriales y territoriales"}

II.1 Producir más, producir mejor: actividades económicas rurales

II.2 Territorios variados, necesidades diversas. Infraestructura y territorios

II.3 Invertir en capital humano: el papel de la educación

II.4 La salud como clave del desarrollo humano

\section{Temas relacionados, objetos de otras políticas nacionales}

III.1 La Estrategia Reforzada de Reducción de la Pobreza

III.2 Seguridad Alimentaria y desarrollo rural: hacia una Política Nacional de Seguridad Alimentaria

III.3 Una cuestión pendiente: la tenencia de la tierra

III.4 Luchando contra la vulnerabilidad: el Programa Nacional de Reducción de Riesgos

La mera lectura de los temas abordados hace ver, por un lado, que era imposible tratarlos de forma exhaustiva en un texto relativamente breve y, por otro lado, pone de manifiesto la clara voluntad de incorporar un enfoque integrado, global y sistémico de los problemas rurales, superando el frecuente escollo del unilateralismo de cualquier género. El mundo rural es complejo y diverso y su verdadero desarrollo sólo será posible mediante un abordaje pluridisciplinar e integrado: he aquí un elemento fundamental de la filosofía del documento de Bases.

Por otro lado se trata de un diagnóstico horizontal, para todo el territorio rural del país, sin entrar en los diagnósticos por grandes áreas que, siguiendo la filosofía de las Bases, es muy necesario dada la diversidad de sus territorios, pero no puede hacerse sin la participación de los agentes locales involucrados en el desarrollo de sus respectivos territorios.

El diagnóstico analítico concluye, de forma más sintética, con un árbol de problemas y una matriz FODA que recoge los aspectos básicos de la problemática rural global del país. 
Por último, el Capítulo Cuarto constituye una primera aportación a la formulación de estrategias y líneas de acción para el desarrollo rural de Nicaragua. Estas estrategias son también un producto fundamental del debate y consenso alcanzados en los Talleres de Selva Negra y del INCH, y han sido completadas con posterioridad a partir de los Principios, del FODA y del conjunto de documentos que entraron en consideración en el capítulo de diagnóstico general.

\section{¿Cuál es su filosofía? Los principios básicos consensuados}

En el apartado de Principios Generales de Partida se enuncian diez principios previos que incluyen los elementos conceptuales, metodológicos y del entorno necesarios para enunciar propiamente los principios inspiradores e instrumentales.

\section{PRINCIPIOS GENERALES DE PARTIDA}

PI I. El desarrollo humano como principio de partida. Un desarrollo rural con equidad en las diferencias.

PI II. Un desarrollo rural humano y sostenible: la compatibilidad con la reproducción de los equilibrios de los ecosistemas.

PI III. La dimensión espacial de las diferencias: la geografía humana.

PI IV. La lucha contra la pobreza en el marco del desarrollo rural.

PI V. La necesidad de un plan estratégico de nación.

PI VI. La importancia del tejido institucional y de la gobernabilidad.

PI VII. Necesidad de estabilidad macroeconómica y crecimiento económico.

PI VIII. El papel crucial de la demografía y las migraciones.

PIIX. La necesidad de un enfoque holístico y sistémico y de una aproximación interdisciplinar.

PI X. Nuevos valores y actitudes en las organizaciones y en los técnicos: Ética del desarrollo y la cooperación. 
Por otro lado, los diez Principios Inspiradores del Desarrollo Rural configuran diez proposiciones de enorme importancia para enmarcar los lineamientos de una eventual política de desarrollo rural. Se inscriben en la línea conceptual del movimiento que ha dado en llamarse "la Nueva Ruralidad Latinoamericana", y constituyen el cuerpo del documento, intentando plantear un marco conceptual claro que oriente la elaboración de estrategias y políticas, planes, programas y proyectos, apuntando enfoques metodológicos de acción sobre el terreno.

PRINCIPIOS INSPIRADORES DEL DESARROLLO RURAL

PII. Un modelo de desarrollo rural endógeno

PI II. Un enfoque centrado en el territorio y su población

PI III. una concepción multifuncional del territorio rural

PI IV. Participación y empoderamiento: Descentralización y Planificación bottom-up o de abajo arriba

PI V. Invertir en capital social

PI VI. Invertir en capital humano

PI VII. Lograr la mayor articulación productiva interna y externa delas respectivas economías territoriales

PI VIII. Coordinación con las políticas macro y sectoriales y entre los actores, los organismos y los agentes de desarrollo. La necesidad de un plan estratégico y una ley de desarrollo rural

PIIX. La gestión del desarrollo rural desde el territorio. Equilibrio, coordinación y partenariado entre sector público y privado. Corresponsabilidad y consenso

PIX. El desarrollo rural como proceso a largo plazo frente al enfoque de proyectos 
¿Cuál es el objetivo y la visión implícitos en las estrategias y propuestas de acción para el desarrollo rural de nicaragua?

\section{Objetivo General}

Mejorar las condiciones de vida de la población rural, ampliando sus posibilidades de opción en los distintos aspectos de la vida en las generaciones presentes y las futuras, en armonía ecológica, con equidad social, de género e intergeneracional, respetando y potenciando los valores culturales propios y la diversidad de etnias y culturas

\section{La Visión}

Los hombres y mujeres que habitan en el campo disfrutan de una calidad de vida tal que les permite desarrollar sus capacidades plenamente $y$ en libertad.

Disfrutan de empleo y de unos ingresos diversos y suficientes, que les permiten mirar al futuro con confianza; de una vivienda segura y digna; de agua potable, luz, saneamiento; de buenas comunicaciones; de servicios de salud y educación de calidad acordes con su realidad para ellos y sus hijos e hijas.

Participan activamente en las decisiones que afectan a su futuro y el de su comunidad, comarca, municipio y territorio.

Su cultura y valores son respetados y potenciados y viven en un entorno natural sano y saludable, donde el cuido y manejo adecuado de sus laderas, valles, aguas y bosques les hacen menos vulnerables a los fenómenos naturales.

Por todo ello deciden permanecer en el campo y no migrar a la ciudad o al extranjero como una elección libre entre otras posibilidades de opción, pues consideran que tienen una mayor calidad de vida permaneciendo en el campo.

Así, el medio rural de Nicaragua constituye un patrimonio de gran valor tanto para sus pobladores como para los habitantes de los núcleos urbanos, donde la pobreza no es un fenómeno generalizado y sí una clase media rural dinámica, y que contribuye mediante el desempeño de sus múltiples funciones, al bienestar de todos los nicaragüenses. 


\section{¿Cuáles son las estrategias, políticas y propuestas más importantes de las bases?}

Aunque han sido discutidas y estructuradas, las estrategias y líneas de acción son meras propuestas para el debate; su principal aportación podría ser abrir una serie de campos de actuación de políticas integradas, sugiriendo en todo momento su adecuación a los problemas diagnosticados y su adecuación a los objetivos de capitalización humana, social, ambiental y productiva. En todo momento se inscriben en el marco más amplio de las necesarias políticas macro de ámbito general y que afecten al país en su conjunto, huyendo de un planteamiento restrictivo puramente sectorial o focalizado.

El resumen de estrategias generales asociadas a problemas se resumen a continuación:

\begin{tabular}{|l|l|}
\hline PROBLEMAS GENERALES (de PAÍs) & ESTRATEGIAS GENERALES (de PAís) \\
\hline P1. Alta proporción de familias con bajos ingresos & $\begin{array}{l}\text { P1. Promover políticas nacionales que fomenten la equi- } \\
\text { dad y la mejor distribución de la riqueza y del ingreso }\end{array}$ \\
\hline P2. Débil entramado institucional & P2. Fortalecer la institucionalidad pública y privada \\
\hline P3. Escasa conexión entre los sistemas rural y urbano & P 3. Articular lo urbano con lo rural \\
\hline P 4. Alta vulnerabilidad frente a desastres naturales & P4. Disminuir la vulnerabilidad ante desastres naturales \\
\hline P5. Degradación del potencial ecológico & P5. Implementar unmanejosostenibledel potencial ecológico \\
\hline P6. Limitado papel de las municipalidades & P6. Fortalecer los gobiernos municipales \\
\hline $\begin{array}{l}\text { P7. Desconexión y descoordinación de las políticas que } \\
\text { afectan al medio rural con las políticas macro y } \\
\text { sectoriales }\end{array}$ & $\begin{array}{l}\text { P7. Coordinar yarticular las políticas macroy las sectoria } \\
\text { les con el desarrollo rural }\end{array}$ \\
\hline
\end{tabular}

\begin{tabular}{|l|l|}
\hline PROBLEMAS HORIZONTALES (RURALES) & ESTRATEGIAS HORIZONTALES (RURALES) \\
\hline $\begin{array}{l}\text { R1. Falta de una política institucional coordinada para } \\
\text { el medio rural }\end{array}$ & $\begin{array}{l}\text { R1. Diseñar y promover una PolíticaNacional deconsenso } \\
\text { del Desarrollo Rural }\end{array}$ \\
\hline R2. Ausencia de un enfoque territorial del desarrollo & R2. Promover un modelo de planificación por territorios \\
\hline R3. Baja inversión en el medio rural & R3. Promover la Inversión en el medio rural \\
\hline $\begin{array}{l}\text { R4. Falta de reconocimiento de la potencialidad y papel } \\
\text { de la mujer rural }\end{array}$ & $\begin{array}{l}\text { R4. Promover el reconocimiento de la potencialidad y } \\
\text { papel de la mujer rural como motor de desarrollo }\end{array}$ \\
\hline $\begin{array}{l}\text { R5. Acceso muy limitado de la población rural a la } \\
\text { educación en sus diferentes niveles }\end{array}$ & R5. Adecuar y fortalecer la Educación Rural \\
\hline $\begin{array}{l}\text { R6. Carencias en la prestación de servicios de salud, } \\
\text { especialmente preocupantes en el medio rural }\end{array}$ & R6. Fortalecer de la atención en salud en las áreas rurales \\
\hline
\end{tabular}




\begin{tabular}{|c|c|}
\hline PROBLEMAS FOCALIZADOS (SECTORIALES) & ESTRATEGIAS FOCALIZADAS (SECTORIALES) \\
\hline $\begin{array}{l}\text { S1. Estructura institucional del sector público agrope- } \\
\text { cuario inexistente o poco operativa en el medio rural }\end{array}$ & $\begin{array}{l}\text { S1. Fortalecer laestructurainstitucional del sector público } \\
\text { agropecuario y forestal }\end{array}$ \\
\hline S2. Baja productividad del sector agropecuario & $\begin{array}{l}\text { S2. Aumentar la productividad del sector agropecuario y } \\
\text { forestal }\end{array}$ \\
\hline S3. Escasa diversificación productiva rural & $\begin{array}{l}\text { S3. Promover ladiversificación productivay defuentes de } \\
\text { ingresos }\end{array}$ \\
\hline $\begin{array}{l}\text { S4. Sector agropecuario desprotegido frente a la } \\
\text { globalización }\end{array}$ & $\begin{array}{l}\text { S4. Mejorar la competitividad de la producción } \\
\text { agropecuaria }\end{array}$ \\
\hline S5. Alto riesgo de la actividad agropecuaria & $\begin{array}{l}\text { S5. Implementar mecanismos que reduzcan el riesgo en } \\
\text { producción agropecuaria }\end{array}$ \\
\hline S6. Escasa integración vertical y horizontal del sector & $\begin{array}{l}\text { S6. Articulación de la producción agropecuaria con los } \\
\text { otros sectores de la economía }\end{array}$ \\
\hline S7. Complejo e incierto sistema de tenencia de la tierra & S7. Ordenamiento y Legalización de la propiedad rural \\
\hline
\end{tabular}

\section{¿Qué significa la adhesión al proceso?}

En el proceso se ha puesto de manifiesto la existencia de un notable consenso de personas e instituciones acerca de los siguientes puntos:

- El interés de todos los participantes en los problemas nacionales.

- La negociación de ideas y análisis interdisciplinario del desarrollo rural.

- La motivación para salir al paso de aquello que nos separa y encontrar mayores puntos de coincidencia teóricos, metodológicos y de acción.

- La voluntad de centrarse en el desarrollo rural integral, trascendiendo visiones meramente técnicas, metodológicas, conceptuales y prácticas, para llegar al interés central: el desarrollo de la gente, de su calidad productiva, ambiental y de vida.

La adhesión al proceso no significa que se asuman todos los detalles del documento sino que existe una voluntad de sumarse a este proceso participativo y comprometerse con el mismo, con el respeto debido a la diversidad de intereses en juego, compartiendo los puntos anteriormente enunciados y la meta del proceso. 
Por otro lado, en Selva Negra se llegó a un amplio consenso acerca de los principios del Desarrollo Rural, tanto en su expresión general de partida, como en cuanto a los Principios que marcan la inspiración o enfoque del Desarrollo Rural de Nicaragua.

Las instituciones y personas que se adhieren al proceso comparten, en lo fundamental, los principios generales e inspiradores enunciados y se comprometen a colaborar desde sus respectivos ámbitos de actuación, y el respeto a la diversidad de actores, en la consecución de un desarrollo rural de esas características

Se podría resumir el contenido material de dichos consensos de la forma siguiente:

\section{En cuanto a los Principios Generales:}

Un desarrollo humano con equidad y sostenible, que tiene su centro en las personas, asumiendo la dimensión espacial de las diferencias, que enfrenta la pobreza pero con visión de largo alcance, que requiere un plan de nación, se apoya en un tejido institucional y en la gobernabilidad, en la estabilidad macroeconómica y el crecimiento económico, en el que la demografía y las migraciones ocupan un rol importante, para el que son imprescindibles unos nuevos valores y actitudes en las organizaciones y las personas, con enfoque de proceso, sistémico y holístico.

2. En cuanto a los Principios Inspiradores del Desarrollo Rural:

Que permiten ver el desarrollo rural como un proceso endógeno, centrado en el territorio y su población, de tipo multifuncional, que implica la participación y el "empoderamiento" de la población, que requiere invertir en capital social y humano y que necesita lograr articulación productiva interna y externa, se coordina con las políticas macro y sectoriales, que imprime equilibrio y coordinación entre lo público y privado y que supone corresponsabilidad y consenso, y que ha de tener una mirada de largo plazo trascendiendo el enfoque de proyectos. 
En relación con el diagnóstico general y con las estrategias (capítulos 30y 4 o, respectivamente de las Bases) el consenso alcanzado se refiere más a la orientación general del documento que a todos y cada uno de los detalles del diagnóstico 0 de las estrategias propuestas. Estas últimas, en particular, habrán de constituir una parte esencial de los siguientes pasos a dar para avanzar en la elaboración de un verdadero Plan Nacional para el desarrollo rural de Nicaragua.

\section{¿Qué queda por hacer?}

La publicación y difusión de las Bases no supone el final del proceso descrito: precisamente se insiste en que el propio proceso es más importante que el documento. Haciendo honor al subtítulo (“Una propuesta para el debate y la acción") se trata en realidad de un comienzo, de una invitación a un debate con todas aquellas personas, entidades o instituciones políticas y de la sociedad civil que trabajan en pro de un futuro mejor para nuestro país $y$, en particular, de su rico y variado mundo rural y, sobre todo, con los habitantes de dicho medio rural. El desarrollo en general, y el desarrollo rural en particular, constituyen ante todo un proceso; para que ese proceso sea exitoso se requiere la participación sinérgica de cuantos actores puedan aportar algo al mismo. Es urgente pensar en el largo plazo.

Por ello, el texto "Bases para un Plan de Desarrollo Rural de Nicaragua. Una propuesta para la discusión y la acción" aspira a provocar la reflexión y el diálogo constructivo. Esperamos, por lo tanto, que la publicación del documento sea el comienzo de un proceso participativo más amplio, que incluya la incorporación al debate y a la acción a la sociedad civil, los organismos del gobierno central, las municipalidades, la comunidad donante y los partidos políticos.

Managua, Septiembre de 2001 